\title{
Reviewer Acknowledgements for Global Journal of Health Science, Vol. 9, No. 4
}

Global Journal of Health Science wishes to acknowledge the following individuals for their assistance with peer review of manuscripts for this issue. Their help and contributions in maintaining the quality of the journal are greatly appreciated.

Global Journal of Health Science is recruiting reviewers for the journal. If you are interested in becoming a reviewer, we welcome you to join us. Please find the application form and details at http://recruitment.ccsenet.org and e-mail the completed application form to gjhs@ccsenet.org.

\section{Reviewers for Volume 9, Number 4}

\begin{tabular}{|c|c|c|}
\hline Abiodun Adeniran & Hesham Atef Abdel Halim & R. Rajkumar \\
\hline Aljameel Albandary & Hugh S. Paterson & Raildo da Silva Coqueiro \\
\hline Amy Clements-Cortes & Ibrahim G. Castro-Torres & Raphaële GIRARD \\
\hline Aris Gkoulalas-Divanis & Jamal Shams & Raymond Jagessar \\
\hline Ayesha Johnson & James Ajigasokoa Ndako & Ryosuke Tsuruta \\
\hline B. Kollia & Juan A. Tovar & Samir Othman \\
\hline Carlos Martin Ardila & Juan Vázquez Estévez & Sara Melo \\
\hline Celia Moffat Joel Matyanga & Kinley Wangdi & Sevgi Turan \\
\hline Chung-Yu Chen & Liu Liu & Shozab Ali \\
\hline Ekta Dahiya & Marisa C R Fonseca & Snehal Padhye \\
\hline Elizabeth Claydon & Mehmet Çinar & Sushma Sagar \\
\hline Esteban Ortiz-Prado & Mini Sood & Tan Ching Siang \\
\hline Evanthia Sakellari & Minjung Seo & Tatsuro ISHIZAKI \\
\hline Faik Ardahan & Mohamed A. Al-Kamel & Ví ctor Javier Lara-Díaz \\
\hline Farahnaz Amini & Mohammad Al-Qudah & Xiaosun Lu \\
\hline Fatih Inci & Monia Ouederni & Yan Ma \\
\hline Francis Lam & Pavlos Sarafis & Yasam Kemal Akpak \\
\hline Francisco Rigla & Pedram Iranmanesh & Yasuhiko Kamada \\
\hline Gabriele Messina & Petros Galanis & Zhen Luo \\
\hline Georgann Weissman & Phillip Austin & \\
\hline Hanna Kische & Polly Yeung & \\
\hline Heba Aboel Naga & Pradnya KAKODKAR & \\
\hline
\end{tabular}

